\title{
Seamless Channel Spacing in Multiple Wavelength Fiber based Lasers
}

\author{
M. Ajiya ${ }^{1 *}$ J. A. Oladapo ${ }^{1}$, U. G. Danbatta ${ }^{2}$, and N. A. M. Ahmad Hambali ${ }^{3}$
}

\begin{abstract}
The spacing between channels in multiple wavelength Brillouin based fiber lasers are inherently $0.08 \mathrm{~nm}$ $(10 \mathrm{GHz})$. We demonstrate multiple - wavelength Brillouin/Erbium fiber laser in the Long wavelength band where the spacing between channels can seamlessly be achieved by changing the values of interferometer delay inserted within the laser cavity. The experimental setup is simulated in Optisystem environment. When the time delay of the interferometer was set at $0.1 \mathrm{~ns}$, spacing between channels stood at the usual $0.08 \mathrm{~nm}$. When the time delay was adjusted to $0.01 \mathrm{~ns}$, the spacing between the generated channels stood at $0.8 \mathrm{~nm}$. The demonstrated laser mitigates the problem associated with fixed channel spaced laser structures.
\end{abstract}

Keywords - Brillouin Fiber Laser, Delay, Interferometer, Stimulated Brillouin scattering, Wavelength Channel Spacing.

\section{INTRODUCTION}

$\mathrm{M}$ ULTI-wavelength fiber lasers have attracted remarkable research interest due to its wide applications in optical communications [1-4]. Notable architecture for the generation of multi-wavelength sources in fiber lasers is what has come to be known as Multi-wavelength Brillouin - Erbium Fiber laser (MWBEFL). MWBEFL was first reported by Cowle and Stepanov in 1996 [5]. It is obtained by in integration of linear gain obtained from Erbium doped fiber (EDF) and nonlinear Brillouin gain obtained from stimulated Brillouin scattering (SBS) effect in optical fiber. Since the introduction of MWBEFL, various types of laser cavity have been reported [68]. The spacing between channels in silica based optical MWBEFL is inherently $10 \mathrm{GHz}(0.08 \mathrm{~nm})$ because of the Doppler effects (change in frequency and wavelength of a wave for an observer moving relative to the source of the waves) [9]. However practical contribution of MBEFL in a practical optical communication system implementation is limited due to the difficulty of channel multiplexing from the narrow $(10 \mathrm{GHz})$ spacing. Therefore attempts have been made to increase the spacing between channels so that multiplexing and de-multiplexing can be achieved with ease in MBEFL. Several works have been reported where double Brillouin

\footnotetext{
${ }^{1}$ Department of Electrical Engineering, Faculty of Engineering, Bayero University, 3011, Kano, Nigeria

${ }^{2}$ Nigerian Communications Comsission, Plot 423, Aguiyi Ironsi Street, Maitama, Abuja, Nigeria.

${ }^{3}$ Semiconductor Photonics \& Integrated Lightwave Systems (SPILS),Tun Abdul Razak Laser Laboratory (TAReL),School of Microelectronic Engineering,Universiti Malaysia Perlis,Pauh Putra Main Campus,02600, Arau, Perlis, Malaysia.
}

frequency $(20 \mathrm{GHz}$ or $0.16 \mathrm{~nm})$ has been achieved in MWBEFL [10-12].

In this paper, we demonstrate an MWBEFL in the $\mathrm{L}-$ band region that utilizes delay interferometer filter in its lasing cavity. By simply changing the time of delay in the interferometer, we discovered that flexibility in channel spacing is obtained. When the time delay of the interferometer was set at $0.1 \mathrm{~ns}$, spacing between channels stood at the usual $0.08 \mathrm{~nm}$. When the time delay was adjusted to $0.01 \mathrm{~ns}$, the spacing between the generated channels stood at $0.8 \mathrm{~nm}$. The experimental setup is simulated in Optisystem environment, a comprehensive software design suite for photonics provided by Optiwave Systems Inc.

\section{SimUlation SET - UP}

The simulation set up of the L-Band Brillouin Erbium fiber laser utilizing Delay interferometer for channel spacing is depicted in Figure1. The set up consist of continuous wave laser at a wavelength of $1600 \mathrm{~nm}$ and power of $-47 \mathrm{dBm}$, Erbium doped fiber amplifier (EDFA) with a gain of $65 \mathrm{~dB}$, delay interferometer at wavelength of $1600 \mathrm{~nm}$, optical circulator, loop control, ideal isolator, single mode fiber (SMF) of $25 \mathrm{~km}$ length, attenuation of $25 \mathrm{~dB}, 16.75 \mathrm{ps} / \mathrm{nm} / \mathrm{km}$ of dispersion and optical spectrum analyzer (OSA) with resolution bandwidth of $0.01 \mathrm{~nm}$. The circuit is implemented in Optisystem, optical system simulation software. The continuous wave laser amplified by the EDFA is used as Brillouin pump (BP). In order to experience SBS effect, a higher BP power is required. The amplified signal is directed to the Delay Interferometer. The signal passes into the circulator and then, it is guided into the SMF. Then the cavity is formed. When the total gains in the cavity, (i.e. Brillouin gain and EDFA gain) are equal to the cavity loss, lasing action starts. The main purpose of EDFA gain is to compensate for the loss in the laser cavity so that SBS effect can be initiated and also to increase the laser output power. The lasing continues, provided the gain in the cavity is equal or greater than the cavity loss. The channel spacing between the Stokes and anti-Stokes is determined by the delay of the Interferometer. The laser's output is measured by Optical Spectrum Analyzer (OSA) connected to the Isolator and Circulator. The output signal measured after isolator is referred to as transmitted signal while the output signal at port 3 of circulator is termed reflected signal. 


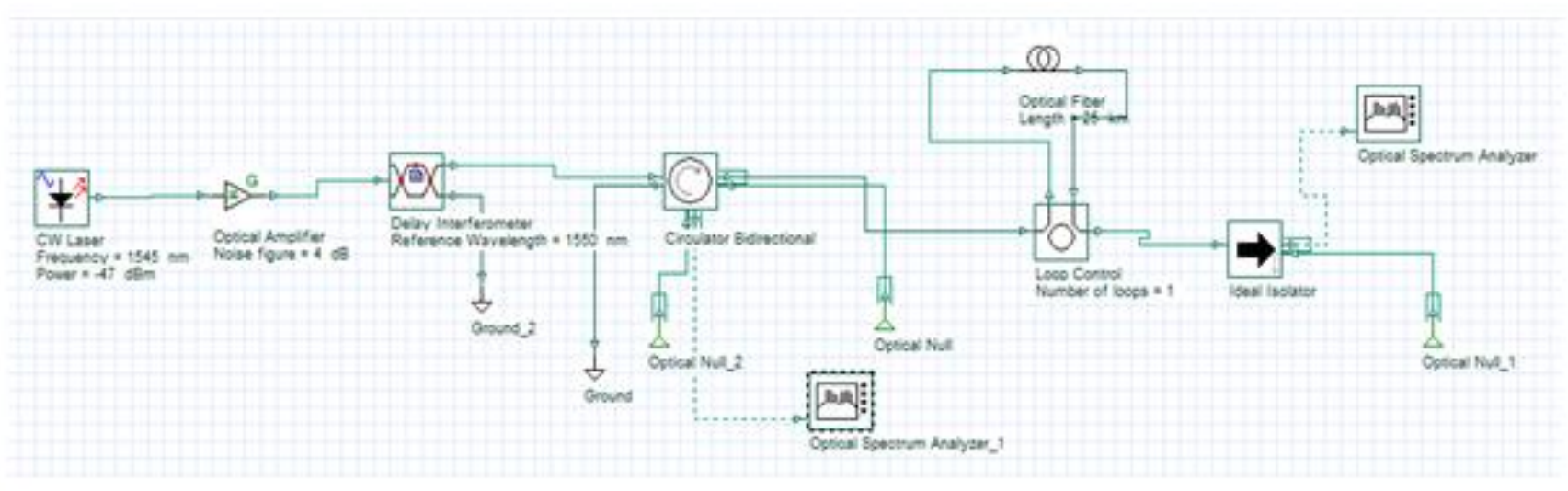

Fig. 1 Simulation Set-Up of the MWBEFL Assisted by Interferometer Delay

\section{RESULTS AND DISCUSSIONS}

Channel spacing as well as the number of output channels produced by L-Band MBEFL utilizing delay interferometer filter was first studied. The time of delay in the interferometer filter is varied from $0.01 \mathrm{~ns}$ to $0.1 \mathrm{~ns}$. It should be noted that at double transmission trip (time delay) which is equivalent to two cascaded delay interferometer, the channel spacing between two adjacent lasing lines reduces. Therefore, multiple transmission trips (time delay) through delay interferometer will further reduce the wavelength spacing and increase the number of output channels [13]. The variation of time delay of interferometer filter with the Channel spacing produced (wavelength spacing between two adjacent lasing lines at different time delay of the interferometer) is given in Figure 2. At 0.025 ns delay, wavelength spacing between two adjacent transmission peaks is $0.33 \mathrm{~nm}$ while the adjacent wavelength spacing between two transmission peaks is $0.17 \mathrm{~nm}$ at $0.05 \mathrm{~ns}$ of time delay of interferometer filter. The reason being that the delay Interferometer acts as a comb filter which makes the wavelengths that match the peaks of the comb oscillates when it is in laser cavity. The wavelength spacing between two adjacent transmission peaks is $0.33 \mathrm{~nm}(\mathrm{t}=0.025 \mathrm{~ns})$ for one transmission trip.

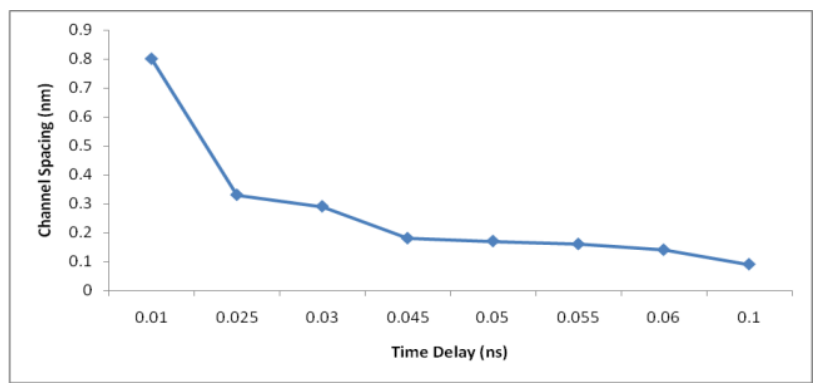

Fig. 2: Wavelength channel spacing against Time delay of the Interferometer.

For the double transmission trip which is equivalent to two cascaded delay interferometer, channel spacing between two lasing lines is reduced. Therefore multiple transmission trips through delay interferometer will further reduce the wavelength spacing and increase the number of output channels.

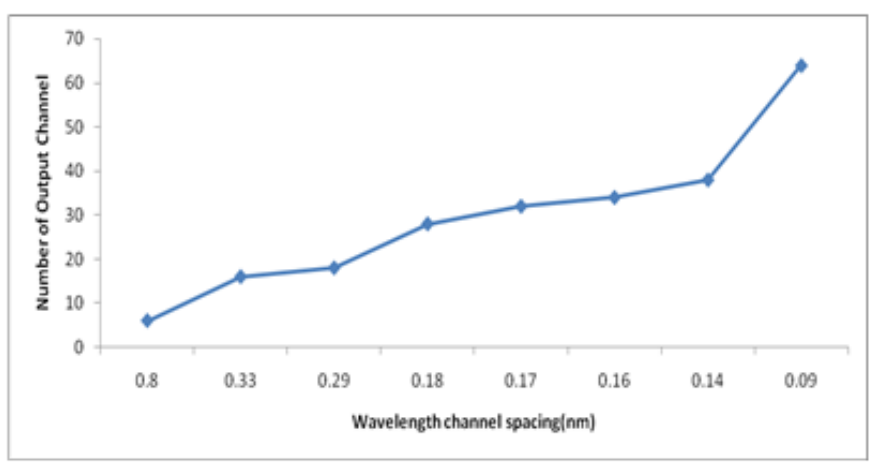

Fig. 3: Output Channels generated against wavelength channel spacing

In Figure 3, total number of generated output channels against channel spacing is presented. It can be seen from Figure 3 that at channel spacing of $0.08 \mathrm{~nm}$, total of 64 output channels comprising of both Stokes and Anti-Stokes signals are generated while at $0.33 \mathrm{~nm}$ channel spacing only 16 output channels are produced.

The output spectra of the MWBEFL at EDFA gain, Brillouin wavelength and Brillouin power of $65 \mathrm{~dB}, 1600 \mathrm{~nm}$ and $-47 \mathrm{dBm}$ respectively with selected delay time in the range of $0.01 \mathrm{~nm}$ to $0.1 \mathrm{~nm}$ are shown in Figures $4 \mathrm{a}-\mathrm{d}$. These Figures clearly shows the wavelength channel spacing and the number of output channel generated from the demonstrated MWBEFL. It can be clearly seen from figure 4 that as the transmission trip (time delay) is increasing, the spacing between adjacent channels reduces but with increase in number of output channels and vice versa. At the maximum time delay of $0.1 \mathrm{~ns}$ about 64 output channels with $0.08 \mathrm{~nm}$ spacing between adjacent channels were obtained while the least time delay of $0.01 \mathrm{~ns}$ produced only 6 output channels with $0.8 \mathrm{~nm}$ spacing between adjacent channels. Therefore, a trade - off exists. 
룰 Time delay $=0.01 \mathrm{~ns}$ Channel spacing $=0.8 \mathrm{~nm}$ Dbl Click On Objects to open properties. Move Objects with Mouse Drag

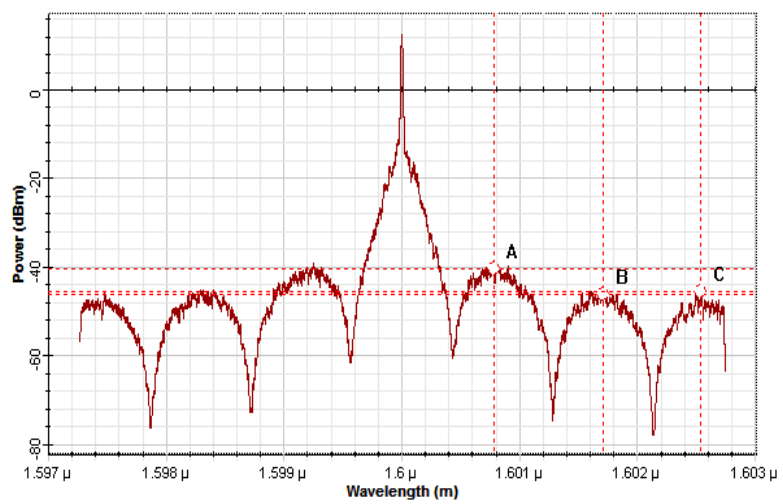

Fig. 4(a)

룰 Time delay $=0.025 \mathrm{~ns}$ Channel spacing $=0.33 \mathrm{~nm}$ Dbl Click On Objects to open properties. Move Objects with Mouse Drag

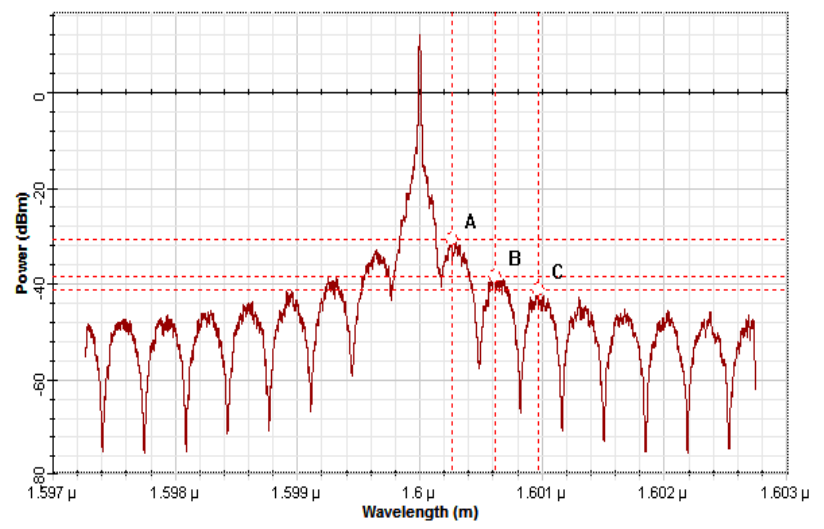

Fig. 4(b)

물 Dbl Click On Objects to open properties. Move Objects with Mouse Drag

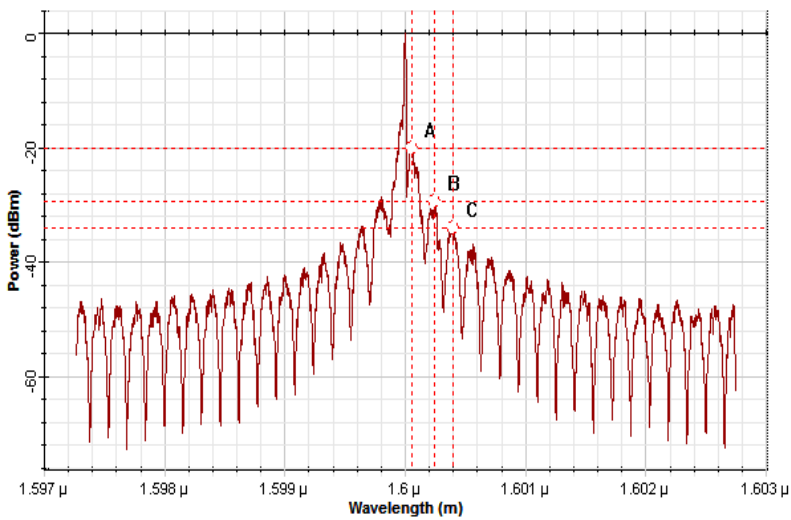

Fig. 4(c)
를 Time delay $=0.1 \mathrm{~ns}$ Channel spacing $=0.08 \mathrm{~nm}$ Dbl click On Objects to open properties. Move Objects with Mouse Drag

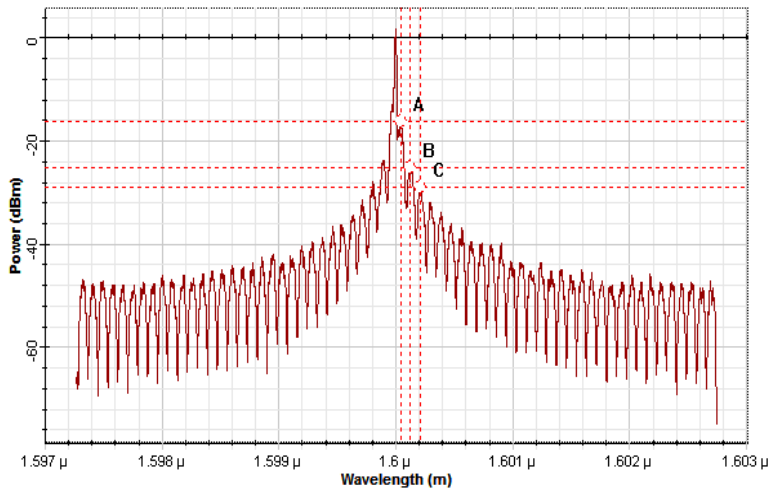

Fig. 4(d)

Fig. 4: Output spectrum of wavelength Channel spacing and number of output channel generated at different Time Delay of Interferometer Filter

\section{CONCLUSION}

Multi-wavelength Brillouin Erbium fiber laser that utilizes delay interferometer as a comb filter has successfully been demonstrated. The laser system is simulated in the Optisystem environment. The influence of time delay and its effect on the generation of output channels in the demonstrated MBEFL was reported. When the demonstrated laser system was set at the standard spacing between channels of $0.08 \mathrm{~nm}, 64$ output channels are generated. However, when the spacing between channels was set to $0.8 \mathrm{~nm}$, only 6 output channels are produced. The demonstrated laser provides a seamless spacing between lasing channels in fiber based lasers thereby providing solutions against channel multiplexing due to narrow spacing between lasing channels.

\section{REFERENCES}

[1] S. W. Harun, X. S. Cheng, P. Poopalan, and H. Ahmad, "Self-excited brillouin-erbium fiber laser for DWDM applications," Optics and Laser Technology, vol. 39, pp. 94-97, 2007.

[2] Y.-J. Kim, B. J. Chun, Y. Kim, S. Hyun, and S.-W. Kim, "Generation of optical frequencies out of the frequency comb of a femtosecond laser for DWDM telecommunication," Laser Physics Letters, vol. 7, pp. 522 $527,2010$.

[3] L. Talaverano, S. Abad, S. Jarabo, and M. Pez-Amo, "Multiwavelength fiber laser sources with Bragg-grating sensor multiplexing capability," Journal of Lightwave Technology, vol. 19, p. 553, 2001.

[4] R. Slavik and S. LaRochelle, "Multiwavelength single-mode erbium doped fiber laser for FFH-OCDMA testing," in Optical Fiber Communications Conference, Anaheim, California United States, 2002, pp. 245-246.

[5] G. J. Cowle and D. Y. Stepanov, "Multiple wavelength generation with Brillouin/erbium fiber lasers," IEEE Photonics Technology Letters, vol. 8, pp. 1465-1467, 1996.

[6] N. Ahmad Hambali, M. Ali Toor, Z. Yusoff, and M. Ajiya, "L-band multi-wavelength Brillouin/Raman fiber laser utilizing the reverse-Sshaped section," Journal of Nonlinear Optical Physics \& Materials, vol. 23, pp. 1450026-10, 20142014.

[7] M. N. Mohd Nasir, Z. Yusoff, M. H. Al-Mansoori, H. A. Abdul Rashid, and P. K. Choudhury, "Broadly tunable multi-wavelength Brillouinerbium fiber laser in a Fabry-Perot cavity," Laser Physics Letters, vol. 5, pp. 812 - 816, 2008.

[8] M. N. M. Nasir, Z. Yusoff, M. H. Al-Mansoori, H. A. A. Rashid, and P. K. Choudhury, "Low threshold and efficient multi-wavelength 
Brillouin/erbium fiber laser incorporating a fiber Bragg grating filter with intra-cavity pre-amplified Brillouin pump," Laser Physics Letters, vol. 6, pp. 54 - 58, 2009.

[9] G. S. He and S. H. Liu, Physics of Nonlinear Optics. Singapore: World Scientific, 1999.

[10] S. W. Harun, M. R. Shirazi, and H. Ahmad, "A new configuration of multi-wavelength Brillouin fiber laser," Laser Physics Letters, vol. 5, 2008.

[11] Y. Shee, M. Al-Mansoori, A. Ismail, S. Hitam, and M. Mahdi, "Multiwavelength Brillouin-erbium fiber laser with double-Brillouinfrequency spacing," Optics Express, vol. 19, pp. 1699-1706, 2010.

[12] N. A. Hambali, M. Al-Mansoori, M. Ajiya, A. A. A. Bakar, S. Hitam, and M. Mahdi, "Multi-wavelength Brillouin-Raman ring-cavity fiber laser with 22-GHz spacing," Laser Physics, vol. 21, pp. 1656-1660, 2011.

[13] K. Suganya and D. S. Sundar, "Hybrid Gain Medium used in Multiwavelength Ring Laser for Noise Reduction," International Journal of Computer Applications, vol. 67, pp. 1 - 4, 2013. 